\title{
An Evaluation of the Studies on the Therapeutic Effects of Yoga in People with Dementia
}

\author{
Authors: \\ Anastasia Bougea' \\ 1. Department of Neurodegenerative Disease, Eginition Hospital, National and \\ Kapodistrian University of Athens, School of Medicine, Athens, Greece \\ *Correspondence to annita139@yahoo.gr \\ Disclosure: \\ The author has declared no conflicts of interest. \\ Received: \\ 06.03 .20 \\ Accepted: \\ 11.05 .20 \\ Citation: \\ EMJ Neurol. 2020;8[1]:64-66
}

\section{INTRODUCTION}

The global population is ageing, and the prevalence of dementia is increasing. Current conventional drug therapy lacks efficacy; therefore, there is growing interest in nonpharmacological interventions of complementary and alternative medicine., ${ }^{1,2}$ Yoga is an ancient Indian practice which is characterised as a mind-body medicine modality according to the National Center for Complementary and Integrative Health $(\mathrm{NCClH}){ }^{1}$ Yoga therapy is an inherently holistic approach including asana (the physical posture), pranayama (breathing exercise), meditation, and guided imagery. Yoga has many therapeutic benefits for individuals with a broad range of chronic diseases such as cancer, asthma, diabetes, arthritis, fibromyalgia, anxiety, and depression. Various physical postures, such as yogic breathing (voluntary control of abdominal breathing) and meditation yoga exercises, stretch and relax muscles while other exercises balance the body and mind to reach a state of mind-body integration. ${ }^{1}$ Many programmes for people with dementia combine yoga with physical and occupational therapy including taichi and dance in community settings based on specific techniques: a) repetition with variations, b) progressive and functional movements, c) step-by-step instructions, d) goal orientation, e) physical care, mindfulness, and breathing, and f) social interaction. ${ }^{1}$ By integrating mindfulness into dementia practice, yoga can promote calmness and improve balance, mobility, and strength for patients.

Although yoga has been used as a complementary health approach for enhancing wellness and physical health, little is known about the impact of yoga on cognitive function in adults with dementia. So far there are only two systematic reviews. In 2017, Du and $\mathrm{Wei}^{3}$ conducted a systematic review of the therapeutic effects of yoga in individuals with dementia, which included one related study, ${ }^{2}$ and found that yoga significantly improved cognitive and motor functions and behavioural issues. ${ }^{3}$ In 2018, Brenes et al. ${ }^{4}$ reviewed four yoga intervention studies of dementia patients with beneficial effects on cognitive domains, particularly attention and verbal memory (Table 1); ${ }^{4}$ however, these conclusions should be considered with caution.

\section{LIMITATIONS OF THE INCLUDED STUDIES}

The first critical issue is the methodology to establish the diagnosis of dementia. Most studies examined mild-to-moderate dementia based on the Mini-Mental State Examination (MMSE) $^{2,5}$ or Short Portable Mental Status Questionnaire (SPMSQ). ${ }^{6}$ 
Table 1: Summary of yoga intervention studies for individuals with dementia in two systematic reviews.

\begin{tabular}{|c|c|c|c|c|}
\hline & Fan, Chen² 2011 & Litchke et al. ${ }^{6} 2014$ & Paller et al. ${ }^{10} 2015$ & Barnes et al..$^{5} 2015$ \\
\hline Study type & Quasi-experimental & Quasi-experimental & Quasi-experimental & Quasi-experimental \\
\hline Sample size (n) & 68 & 19 & 17 & 12 \\
\hline Participants & $\begin{array}{l}\text { Mild-to-moderate } \\
\text { dementia }\end{array}$ & $\begin{array}{l}\text { Mild-to-severe } \\
\text { Alzheimer's disease }\end{array}$ & $\begin{array}{l}\text { Mild cognitive } \\
\text { impairment, } \\
\text { Alzheimer's disease, } \\
\text { frontotemporal } \\
\text { dementia, subjective } \\
\text { cognitive complaints }\end{array}$ & Dementia \\
\hline Control group & NA & NA & NA & Usual care \\
\hline $\begin{array}{l}\text { Yoga programme } \\
\text { (duration, frequency/ } \\
\text { week) }\end{array}$ & $\begin{array}{l}\text { Silver yoga/hatha (55 } \\
\text { min, 3x/12 weeks) }\end{array}$ & $\begin{array}{l}\text { Lakshmi Voelker Chair } \\
\text { Yoga } \\
\text { ( } 60 \text { min, 3x/10 weeks) }\end{array}$ & $\begin{array}{l}\text { Gentle yoga, loving- } \\
\text { kindness practice, } \\
\text { meditation } \\
\text { (1.5 hours, 1x/8 weeks) }\end{array}$ & $\begin{array}{l}\text { Body awareness, } \\
\text { chair-based exercises } \\
\text { adapted from tai-chi, } \\
\text { yoga, and Feldenkrais } \\
\text { (45 min, 3x/18 weeks) }\end{array}$ \\
\hline Adherence (\%) & 90.9 & 70.3 & NA & 72.2 \\
\hline $\begin{array}{l}\text { Cognitive } \\
\text { measurements }\end{array}$ & MMSE & SPMSQ & $\begin{array}{l}\text { RBANS } \\
\text { Trail Making Test Parts } \\
\text { A and B }\end{array}$ & MMSE, ADAS-Cog \\
\hline Biomarkers & No & No & No & No \\
\hline Adverse events & NA & NA & NA & $\begin{array}{l}\text { Dizziness/nausea, legs } \\
\text { buckling, hip pain }\end{array}$ \\
\hline Main outcomes & $\begin{array}{l}\text { Improvement of } \\
\text { neuropsychiatric } \\
\text { and physical } \\
\text { symptoms }\end{array}$ & $\begin{array}{l}\text { No significant } \\
\text { improvement of } \\
\text { cognitive symptoms } \\
\text { and activities of daily } \\
\text { living }\end{array}$ & $\begin{array}{l}\text { Improvement of } \\
\text { neuropsychiatric, } \\
\text { cognitive, and physical } \\
\text { symptoms and quality } \\
\text { of life }\end{array}$ & $\begin{array}{l}\text { Improvement of } \\
\text { cognitive symptoms, } \\
\text { caregiver distress, and } \\
\text { quality of life }\end{array}$ \\
\hline
\end{tabular}

ADAS-Cog: Alzheimer's Disease Assessment Scale-Cognitive Subscale; MMSE: Mini-Mental State Examination; NA: not available; RBANS: Repeatable Battery for the Assessment of Neuropsychological Status; SPMSQ: Short Portable Mental Status Questionnaire.

However, the content of the MMSE is highly verbal, lacking sufficient items to adequately measure visuospatial and/or constructional praxis. Litchke et al. ${ }^{6}$ used the SPMSQ as a sensitive and specific screening test for moderate-to-severe dementia; therefore, it should not serve as the sole criterion for dementia diagnosis or for differentiating between various forms of dementia. ${ }^{7}$ Furthermore, the included studies did not use any specific clinical criteria for dementia subtyping. According to the updated criteria for the diagnosis of Alzheimer's disease, ${ }^{8}$ to better characterise these patients, neuroimaging data of genetic and blood/ cerebrospinal fluid (amyloid and tau) biomarkers are required to support the diagnosis of dementia in future studies. ${ }^{9}$
A second limitation is the lack of specific neuropsychological measurements to detect changes in different cognitive domains. ${ }^{2}$ In the study of Paller et al., ${ }^{10}$ patients with dementia were assessed based on the Repeatable Battery for the Assessment of Neuropsychological Status (RBANS) for each of the five domains tested (immediate memory, visuospatial/ constructional, language, attention, and delayed memory) and Trail Making Test (TNT) (Part A [processing speed]). ${ }^{10}$ There was no assessment of executive function, phonemic fluency, or motor responses. ${ }^{10}$ Barnes et al. ${ }^{5}$ used the Alzheimer's Disease Assessment Scale-Cognitive Subscale (ADAS-Cog) that includes direct assessment of learning (word list), naming (objects), following commands, constructional praxis (figure copying), ideational praxis (mailing a letter), orientation 
(person, time, place), recognition memory, and remembering test instructions. Measurement of these neuropsychological variables at multiple time points would have been more informative as cognitive domains can have differential trajectory of change. Additionally, the authors did not use any other tests to assess quality of life or anxiety, which may affect cognition and mental wellbeing before and after the yoga intervention. ${ }^{2,5,6}$ Finally, it would be more appropriate to combine neuropsychological and fluid biomarker data to accurately measure the cognitive changes produced by yoga programmes, as was previously suggested for other neurodegenerative disease (e.g., Parkinson's disease). ${ }^{11}$

Third, there are several factors that may limit the generalisability of the above studies. The study sample size was small, which limited the applicability of the results to a broader population. In all studies, expectation bias may have existed because participants were aware of the treatment allocation. In both studies, participants were enrolled through convenience sampling, thus, selection bias should also be considered. In the study of Fan and Chen, ${ }^{2}$ possible differences in characteristics might have existed that were not captured in this study including a cluster effect. There might have been a potential bias based on dropout rate $(n=33)$, particularly with the moderate sample size, ${ }^{2}$ and furthermore, the randomisation was not entirely perfect in the included studies resulting in a female predominance. In future studies, an experimental design with three groups (yoga group, standard exercise group, and control group) using preand post-test comparisons should be applied. Furthermore, the results of these reviews were based on a unique population of people with mild-to-moderate dementia who attended short follow-up sessions.

Finally, there is wide variability in the type and method of yoga used for intervention and few studies report the training type/level of their instructors. While this is reflective of the fact that there are several different types of yoga disciplines and practices, it limits the generalisability of results. Frequency and intensity (hours, weeks) of intervention periods generally fell within the 8-12week range. This variability of yoga programmes does not permit the monitoring of intervention fidelity and these disparities negatively impact the conclusions on dose-response relationships.

\section{IMPLICATIONS FOR FUTURE STUDIES}

More consistent documentation of the intervention, instructor training, and frequency and intensity will elevate the quality of yoga research. There is a need for future multicentre yoga studies to incorporate a multidisciplinary programme including physicians, nurses, social workers, and psychologists. The key message of yoga is to encourage and enable people with dementia to remain physically active for as long as possible so they can reap the many benefits. All health providers may consider recommending yoga to individuals with dementia as a safe and potentially beneficial complementary health approach. Considering that dementia limits the ability to perform a mental programme such as yoga, healthcare professionals should target patients who are in the mild and moderate stages of dementia. The latter allows the proposition of yoga programmes characterised by a simple language, with clear and repetitive instructions, e.g., using imitation as a starting point. Given the lack of mechanistic studies that support the practice of yoga in the context of dementia, these studies are important to elucidate stress-related pathways and improve behavioural mediated disorders in the future.

\section{CONCLUSION}

Overall, the evidence on the effects of yoga in patients with dementia is limited and conflicting in some cases. Although promising, studies that combine yoga have methodological limitations (small sample, absence of biomarkers/follow up, brain MRI, different protocols/measures). Although their effectiveness is rather modest, the absence of serious side effects allow us to encourage their use in addition to standard treatments. Nevertheless, the preliminary nature of the existing evidence highlights the need for longitudinal studies with a rigorous methodology to define the optimal frequency and intervals of yoga, and to evaluate the costeffectiveness for people with dementia. Given these considerations and the increasing availability of yoga in community settings, clinicians may consider recommending yoga to individuals with dementia. Future rehabilitation programmes with yoga could consider integrating more skills (sleep routine, exercise, healthy 


\section{References}

1. Salazar MC et al. Hatha yoga effects on Alzheimer patients (AP). Act Colom Psicol. 2017;20(1):123-38.

2. Fan JT, Chen KM. Using silver yoga exercises to promote physical and mental health of elders with dementia in long-term care facilities. Int Psychogeriatr. 2011;23(8):1222-30.

3. Du Q, Wei Z. The therapeutic effects of yoga in people with dementia: a systematic review. Int J Geriatr Psychiatry. 2017;32(1):118.

4. Brenes GA et al. The effects of yoga on patients with mild cognitive impairment and dementia: a scoping review. Am J Geriatr Psychiatry. 2019;27(2):188-97.
5. Barnes DE et al. Preventing loss of independence through exercise (PLIE): a pilot clinical trial in older adults with dementia. Plos one. 2015;10(2):e0113367.

6. Litchke LG et al. Benefits of chair yoga with mild to severe Alzheimer's disease. Act Adapt Aging. 2012;36(4):317-28

7. Arevalo-Rodriguez I et al. Mini-mental state examination (MMSE) for the detection of Alzheimer's disease and other dementias in people with mild cognitive impairment (MCl). Cochrane Database Syst Rev. 2015;2015(3):CD010783.

8. Dubois B et al. Advancing research diagnostic criteria for Alzheimer's disease: the IWG-2 criteria. Lancet Neurol. 2014;13(6):614-29.

9. Varatharajah $\mathrm{Y}$ et al. Predicting short-term $\mathrm{MCl}$-to-AD progression using imaging, CSF, genetic factors, cognitive resilience, and demographics. Sci Rep. 2019;9(1):2235

10. Paller KA et al. Benefits of mindfulness training for patients with progressive cognitive decline and their caregivers. Am J Alzheimer's Dis Other Demen. 2015;30(3):257-67.

11. Cheung $C$ et al. Effects of yoga on oxidative stress, motor function, and non-motor symptoms in Parkinson's disease: a pilot randomized controlled trial. Pilot Feasibility Stud. 2018;4:162. 